\title{
A multiscale crack path predicting computational method for laminated fiber reinforced composites
}

\author{
Siva Shankar Rudraraju, Ryan Vignes*, Amit Salvi†, Krishna Garikipati $\ddagger$ Anthony M. Waas ${ }^{\ddagger}$ \\ College of Engineering, University of Michigan, Ann Arbor, MI 48109, USA
}

\begin{abstract}
A variational multiscale method (VMM) which incorporates the mechanics of crack growth (displacement discontinuity) in a laminated fiber reinforced composite is implemented within a standard finite element (FE) framework. Traction-separation laws that act across the crack faces are built into the framework and these are activated during the process of crack bridging and advancement. Inputs to the VMM traction-separation laws are obtained by conducting standard fracture mechanics tests (a compact tension test to obtain the fracture energy and a double notched tension test to obtain the cohesive strength). Armed with these inputs, the VMM is verified and validated by conducting simulations and tests on single edge notch three point bend (SEN3B) fracture specimens. The VMM predictions are also compared against traditional discrete cohesive zone method (DCZM) predictions. This comparison reveals the inherent superiority of the VMM approach which requires no a priori knowledge of the crack propagation direction.
\end{abstract}

\section{Introduction}

Displacement discontinuities are abrupt variations in an otherwise smooth displacement field caused due to changes in the local load bearing capacity of the material. Physically, these discontinuities manifest themselves as shear bands, cracks and kink bands in materials. Once initiated, these discontinuities may lead to intense localized deformation and hence ultimate failure of materials. Hence the ability to numerically simulate the evolution of these discontinuities may prove to be of immense help in the fracture analysis of a wide range of materials.

Analyzing the formation of these discontinuities requires working within a multiscale framework. The multiscale approach separates the displacement field into coarse and fine scale components which correspond to the continuous and discontinuous components of the displacement field respectively. This multiscale framework incorporated into the variational equations facilitates the numerical implementation of the VMM methodology in a conventional finite element setting.

While the variational multiscale methodology for simulating displacement discontinuity evolution is well known [Armero 1996, ${ }^{2}$ Armero 1999, ${ }^{3}$ Regueiro 1999, ${ }^{4}$ Borja 2001, ${ }^{5}$ Garikipati $2002^{6}$ ], its application to the prediction of crack growth in laminated fiber reinforced composite materials has not been attempted. In this paper, we present our study of applying the VMM to predicting crack growth in a layered, continuous fiber reinforced (pre-preg based), carbon fiber laminated composite panel.

\section{Continuum Setting}

In this section, the VMM formulation is presented in a very general setting, for a continuum that can entertain a displacement discontinuity. Of interest in this paper is the advancement of a single throughthe-thickness crack like discontinuity in a laminated fiber reinforced panel. Let $\Omega$ denote the domain of the

\footnotetext{
${ }^{*}$ Graduate Student, Department of Mechanical Engineering.

$\dagger^{\dagger}$ Graduate Student, Department of Aerospace Engineering.

$¥$ Associate Professor, Department of Mechanical Engineering.

§Professor, Department of Aerospace Engineering and Department of Mechanical Engineering. Associate Fellow, AIAA.
} 
continuum body (Figure 1) before the development of the displacement discontinuity with particles within the domain labeled $\boldsymbol{x}$. The boundary of the domain is smooth, continuous, and denoted $\partial \Omega$. The boundary and its disjoint subsets, $\partial_{\boldsymbol{u}} \Omega$ and $\partial_{\boldsymbol{\sigma}} \Omega$, are related as

$$
\overline{\partial_{u} \Omega \cup \partial_{\boldsymbol{\sigma}} \Omega}=\partial \Omega, \quad \partial_{u} \Omega \cap \partial_{\boldsymbol{\sigma}} \Omega=\varnothing,
$$

allowing the Dirichlet and Neumann boundary conditions to be specified as

$$
\begin{aligned}
\boldsymbol{u}(\boldsymbol{x}, t) & =\hat{\boldsymbol{u}}(\boldsymbol{x}, t) ; & & \forall \boldsymbol{x} \in \partial_{\boldsymbol{u}} \Omega \\
\boldsymbol{\sigma}(\boldsymbol{x}, t) \overline{\boldsymbol{n}}(\boldsymbol{x}, t) & =\overline{\boldsymbol{T}}(\boldsymbol{x}, t) ; & & \forall \boldsymbol{x} \in \partial_{\boldsymbol{\sigma}} \Omega .
\end{aligned}
$$

In $(2), \hat{\boldsymbol{u}}(\boldsymbol{x}, t)$ is the known displacement specified on $\partial_{\boldsymbol{u}} \Omega, \overline{\boldsymbol{T}}(\boldsymbol{x}, t)$ is the traction prescribed on $\partial_{\boldsymbol{\sigma}} \Omega, \boldsymbol{\sigma}(\boldsymbol{x}, t)$ is the stress, and $\overline{\boldsymbol{n}}(\boldsymbol{x}, t)$ is the unit outward normal to $\partial \Omega$.

As shown in Figure 1, after a displacement discontinuity develops, the body is separated into two regions by a surface, denoted $\Gamma$, across which the displacement field is discontinuous. As the discontinuity propagates through the body, two distinct regions of $\Omega$ emerge. $\Omega_{+}$is the region of the body into which the unit outward normal of the discontinuity surface is directed, while $\Omega_{-}$denotes the remainder of the body. The disjoint subsets of $\Omega$ are related by

$$
\overline{\Omega_{-} \cup \Omega_{+} \cup \Gamma}=\Omega \text {. }
$$

Before the displacement discontinuity develops, the displacement is assumed continuous throughout $\Omega$. The displacement is written as $\boldsymbol{u}(\boldsymbol{x}, t)=\overline{\boldsymbol{u}}(\boldsymbol{x}, t)$ where $\overline{\boldsymbol{u}}(\boldsymbol{x}, t)$ is a continuous function. Once the discontinuity develops, the displacement becomes composed of two parts, a continuous component and a discontinuous component. The total displacement is then written as

$$
\boldsymbol{u}(\boldsymbol{x}, t)=\overline{\boldsymbol{u}}(\boldsymbol{x}, t)+\llbracket \boldsymbol{u} \rrbracket(t) \mathscr{H}_{\Gamma}(\boldsymbol{x}),
$$

where $\llbracket \boldsymbol{u} \rrbracket$ is the magnitude of the discontinuity and is a function of time alone. $\mathscr{H}_{\Gamma}(\boldsymbol{x})$ is the Heaviside function, which locates the discontinuity surface and is defined as

$$
\mathscr{H}_{\Gamma}(\boldsymbol{x})= \begin{cases}0 & \text { if } \boldsymbol{x} \in \Omega_{-}, \\ 1 & \text { if } \boldsymbol{x} \in \Omega_{+} \cup \Gamma .\end{cases}
$$

As the Heaviside function is a function of position alone, the rate of displacement is obtained as

$$
\dot{\boldsymbol{u}}(\boldsymbol{x}, t)=\dot{\bar{u}}(\boldsymbol{x}, t)+\llbracket \dot{\boldsymbol{u}} \rrbracket(t) \mathscr{H}_{\Gamma}(\boldsymbol{x}) .
$$

Restricting the following analysis to infinitesimal displacements, the strain field is obtained as the symmetric gradient of the displacement rate. Using results from the theory of distributions, ${ }^{15}$ the gradient of the Heaviside function is determined as $\nabla \mathscr{H}_{\Gamma}=\boldsymbol{n} \delta_{\Gamma}$, where $\delta_{\Gamma}$ is the Dirac-delta function located at the discontinuity surface and $\boldsymbol{n}$ is the unit normal to the discontinuity surface. Using this result, the strain field is obtained as,

$$
\operatorname{sym}[\nabla \dot{\boldsymbol{u}}]=\operatorname{sym}[\nabla \dot{\overline{\boldsymbol{u}}}]+\operatorname{sym}\left(\llbracket \dot{\boldsymbol{u}} \rrbracket(t) \otimes \boldsymbol{n} \delta_{\Gamma}\right) .
$$

The strain field is then composed of a continuous portion, $\operatorname{sym}[\nabla \dot{\overline{\boldsymbol{u}}}]$, and a discontinuous component, $\operatorname{sym}\left(\llbracket \dot{\boldsymbol{u}} \rrbracket(t) \otimes \boldsymbol{n} \delta_{\Gamma}\right)$. Importantly, in working within the distributional framework, it naturally arises that the discontinuous portion of strain takes the form of a Dirac-delta distribution.

The key aspect in analyzing displacement discontinuities on identifying the distributional characteristics of main quantities, such as the stress, $\boldsymbol{\sigma}$, and internal variables. It can be shown that in a displacement discontinuity problem, the strain field is a singular distribution but the stress and tractions are regular, smooth distributions. The details of the kinematics of the discontinuity, governing equations, distributional analysis $^{6}$ and a constitutive model $^{8}$ that is appropriate for fiber reinforced composites are available in the literature. 


\section{Finite Element Formulation}

\section{A. Galerkin Weak Form}

The weak form of the governing equilibrium equations presented here were developed following the methods presented in [Garikipati 2002 ${ }^{6}$, Borja $2001^{5}$ Regueiro $1999^{4}$. The weak form of the balance of linear momentum is given by:

$$
\int_{\Omega}(\operatorname{sym}[\nabla \boldsymbol{w}]: \boldsymbol{\sigma}) \mathrm{dV}=\int_{\Omega} \boldsymbol{w} \cdot \boldsymbol{b} \mathrm{dV}+\int_{\partial_{\boldsymbol{\sigma}} \Omega} \boldsymbol{w} \cdot \boldsymbol{T} \mathrm{dS}
$$

where $\boldsymbol{T}$ is the traction, $\boldsymbol{b}$ is the body force, and $\boldsymbol{\sigma}$ is the stress. To embed the fine scale physics into the coarse scale weak form, the displacement relation is chosen to be of the form:

$$
\boldsymbol{u}(\boldsymbol{x}, t)=\overline{\boldsymbol{u}}(\boldsymbol{x}, t)+\llbracket \boldsymbol{u} \rrbracket(t) \mathscr{H}_{\Gamma}(\boldsymbol{x}),
$$

where $\overline{\boldsymbol{u}}$ is the continuous displacement and $\llbracket \boldsymbol{u} \rrbracket$ is the discontinuous portion. This choice of the displacement field leads to a multi-scale weak form cast entirely in terms of coarse scale quantities yet still capable of capturing fine scale effects. The displacement field of (9) is now reparameterized as

$$
\boldsymbol{u}(\boldsymbol{x}, t)=\overline{\boldsymbol{u}}(\boldsymbol{x}, t)+\llbracket \boldsymbol{u} \rrbracket(t) M_{\Gamma}(\boldsymbol{x}),
$$

where $M_{\Gamma}(\boldsymbol{x})$ locates the jump across the discontinuity surface, $\llbracket M_{\Gamma} \rrbracket=1$ and has compact support in $\Omega^{\prime h}$ such that $M_{\Gamma}=0$ on $\Gamma_{ \pm}$in Figure $2 . \Omega^{\prime h}$ is the particular finite element through which the discontinuity propagated.

The strain field in an element which has localized and has a discontinuous displacement is then give as

$$
\boldsymbol{\epsilon}_{e}^{h}=\operatorname{sym}\left(\nabla \overline{\boldsymbol{u}}^{h}\right)+\operatorname{sym}\left(\llbracket \boldsymbol{u} \rrbracket_{e} \otimes \boldsymbol{n}\right) \delta_{\Gamma}-\operatorname{sym}\left(\nabla f_{e}^{h} \otimes \llbracket \boldsymbol{u} \rrbracket_{e}\right),
$$

where $e$ denotes a localized element. The first and third terms on the right hand side of (11) are regular components of the strain field while the second term is a singular component. The first term forms the compatible strain field while the remaining two terms are the enhanced contributions which are not subject to any inter-element continuity requirements. The variation and its gradient are expressed in a similar manner,

$$
\boldsymbol{w}=\overline{\boldsymbol{w}}+\llbracket \boldsymbol{w} \rrbracket M_{\Gamma}, \quad \boldsymbol{\beta}_{e}^{h} \equiv \operatorname{sym}\left(\nabla \boldsymbol{w}_{e}^{h}\right)=\operatorname{sym}\left(\nabla \overline{\boldsymbol{w}}_{e}^{h}\right)+\tilde{\boldsymbol{\beta}}_{e}^{h},
$$

where $\overline{\boldsymbol{w}}$ satisfies the usual boundary condition, $\overline{\boldsymbol{w}}=0$, in the region where $\boldsymbol{u}$ is specified.

The weak form of equilibrium then takes on the form ${ }^{7}$,

$$
\begin{aligned}
& \int_{\Omega} \operatorname{sym}\left(\nabla \overline{\boldsymbol{w}}^{h}\right): \boldsymbol{\sigma} \mathrm{dV}=\int_{\Omega} \overline{\boldsymbol{w}}^{h} \cdot \boldsymbol{b} \mathrm{dV}+\int_{\partial_{\boldsymbol{\sigma}} \Omega} \overline{\boldsymbol{w}}^{h} \cdot \boldsymbol{T} \mathrm{dS} \\
& \int_{\Omega_{e}^{\prime}} \tilde{\boldsymbol{\beta}}^{h}: \boldsymbol{\sigma} \mathrm{dV}=0,
\end{aligned}
$$

where $\operatorname{sym}\left(\nabla \overline{\boldsymbol{w}}^{h}\right)=\overline{\boldsymbol{\beta}}$ is the compatible (standard) component of the total strain variation and $\tilde{\boldsymbol{\beta}}$ is the enhanced component. Equation $(13)_{1}$ is the weak balance of linear momentum while $(13)_{2}$ allows the patch test to be satisfied in the presence of an enhanced strain field.

\section{B. Finite Element Discretization}

Two interpolation functions are introduced, $\bar{N}(\boldsymbol{x})$ for the coarse scale, and $N^{\prime}(\boldsymbol{x})$ for the fine scale. With these interpolation functions, the coarse and fine scale displacements are obtained as

$$
\overline{\boldsymbol{u}}^{h}(\boldsymbol{x})=\sum_{A=1}^{n_{\text {node }}} \bar{N}_{A}(\boldsymbol{x}) \boldsymbol{d}^{A} \quad \text { and } \quad \boldsymbol{u}^{\prime h}(\boldsymbol{x})=N^{\prime}(\boldsymbol{x}) \llbracket \boldsymbol{u} \rrbracket .
$$

where $n_{\text {node }}$ are the number of nodes on the element and $\boldsymbol{d}^{A}$ are the associated nodal values of $\overline{\boldsymbol{u}}^{h}(\boldsymbol{x})$. All time dependence has been placed on $\boldsymbol{d}^{A}$ and $\llbracket \boldsymbol{u} \rrbracket$. Figure 3 depicts these shape functions in a one-dimensional setting 


\section{Numerical Implementation for Laminated Composite Panels}

While a natural condition for the emergence of localization (and hence the triggering of the localized deformation computations at the fine scale), has been presented for homogeneous elastic-plastic solids, ${ }^{6}$ conditions for the onset of crack advancement in laminated fiber reinforced panels has been attempted by replacing the laminated non-homogeneous (through-the-thickness) panel by an equivalent homogenized orthotropic panel after which methods of classical fracture mechanics can be invoked. Of interest in the present work are the conditions that would be effective in advancing a through-the-thickness crack like feature that is present in a laminated panel. Studies on this topic are scare. ${ }^{10}$ As a first attempt in predicting crack advancement, we use a critical stress to trigger crack advancement. The critical stress value for crack advancement and hence for triggering the local calculations is termed a material constant and hence is an input to the FE model. This stress is experimentally determined and in conjunction with a VMM simulation of the generating test (in this case the CTS test) is also numerically calibrated so that effects of mesh size and mesh density are understood. Once the local calculations are triggered, the fracture mode (I/II/mixed) of crack propagation is determined based on the local stress state. As the crack advances, softening traction-separation laws provide the necessary bridging tractions, however, unlike in classical cohesive zone methods ${ }^{9,11-14}$ where it is a-priori decided where to place the cohesive elements, in the VMM, the same FEA element acts both as a continuum and also as a cohesive element. This novelty is indispensable in the analysis of crack growth problems where the crack path is not obvious. For the results presented in this paper, a linear softening response has been assumed in both the normal and tangential directions, which in component form can be expressed as,

$$
T_{n}=T_{n_{0}}-\mathcal{H}_{n} \xi_{n}, \quad \text { and } \quad T_{m}=T_{m_{0}}-\mathcal{H}_{m} \xi_{m}
$$

$T_{n}$ is the normal traction on the discontinuity surface, whose maximum value is $T_{n_{0}}$ the moment the localization condition is satisfied. ${ }^{a}$ Similarly, $T_{m_{0}}$ is the maximum tangential traction on the surface when the localization condition is met. $\mathcal{H}_{n}$ is the normal (opening mode) softening modulus and $\mathcal{H}_{m}$ is the tangential (slip mode) softening modulus. $\xi_{n}$ and $\xi_{m}$ are the discontinuity magnitudes in the opening and slip directions. For the assumed linear softening response defined in (15), the softening moduli are obtained from knowing the cohesive strengths and fracture energies which are determined through a combination of experiments and simulations of a double notch tension test and a CTS fracture test, respectively. ${ }^{14}$

The micro-mechanical surface laws govern the crack path within the elements that contain the advancing crack path in the FEA implementation. However, it also important to ensure proper crack propagation from a "parent" element to a subsequent "cracked" element. This is achieved by a crack path tracking algorithm described below.

\section{A. Crack path tracking algorithm}

Figure 4 depicts the crack path tracking algorithm used in the code. Shaded elements have already cracked and are undergoing crack bridging through the softening tractions. The magnitude of the softening is determined by the crack face separation in the element(Figure 5). The crack path (xyy') has reached the edge of the third element and its leading edge is positioned at $y^{\prime}$. The path is pinned there until the third element has reached the condition for crack initiation in a certain load increment. Once the localization criterion is met, the direction of propagation is determined(perpendicular to the maximum principal stress) and its projection on the other element edge $\left(y^{\prime \prime}\right)$ is stored for subsequent crack advancement. Now the crack is propagated through the third element (physically this means the crack tip is now positioned at $y^{\prime \prime}$.

\section{B. FEA Implementation}

An ABAQUS user element (UEL) has been developed based on the VMM framework presented above. The UEL framework allows the developer to essentially create a custom element which incorporates the material model, integration point level computations like element stiffness matrix formation, stress computation and other necessary calculations (static condensation, crack propagation, etc). The global level tasks of assembly and equation solving are performed by the ABAQUS solver. Interested readers are referred to the ABAQUS documentation for detailed information on the UEL framework. At this point it is to be noted that as

\footnotetext{
${ }^{\mathrm{a}}$ It is to be noted that the softening laws are synonymous with the classical cohesive traction separation laws that are used to model crack advancement using traditional cohesive zone methods.
} 
integration point calculations are internal to the UEL and since ABAQUS has no access to this data, it is not possible to develop contour visualizations of the UEL models. We therefore overlaid a dummy mesh of conventional three noded triangles on the three noded UEL element mesh to obtain qualitative contour representations of field variables of interest.

\section{Experimental Study}

The experimental studies conducted to supplement the VMM numerical implementation can be classified into material data input experiments and simulation validation experiments. These experiments were conducted on $[-45 / 0 /+45 / 90]_{6 s}$ laminated fiber reinforced composite specimens cut from large panels.

\section{A. Material data input experiments}

The following data were required as inputs for the FEA implementation.

a) Material data (Elastic Moduli, Yield strength, Plasticity parameter a66 ${ }^{8}$ )

b) Fracture toughness (Mode I/Mode II)

c) Critical cohesive strength (used as the critical localization stress)

The fracture toughness values were extracted from experiments on the standard Compact Tension(CT) specimens (Figure 6). The critical cohesive strength value was backed out from the double notch tension specimen tests (Figure 7).

\section{B. VMM simulation validation experiments}

A series of experiments were carried on standard single edge notch three point bending(SEN3B) specimens with various sizes. The compact tension specimen(CTS) and double notched tension (DNT) specimen provided the input parameters to the VMM calculation, hence the SEN3B experiments were chosen as standalone verification tests for the VMM framework validation.

As shown in Figure 8, the SEN3B specimens were loaded in a three point bend configuration. Extra supports that do not bear any loads were provided to prevent twist buckling in larger specimens. These anti-buckling guides prevent the specimen from displacing out-of the plane of laminate, ensuring no mode III component during crack advancement.

\section{Numerical Simulations}

\section{A. UEL validation - Isotropic material}

Initial studies on simple problems such as cracking of a shear block and tension specimen were simulated to validate the VMM methodology and UEL implementation for isotropic materials. Such implementations for isotropic materials are available in the literature in the context of shear band propagation. ${ }^{1}$ The results from these simple analysis that have been done before were reproduced in order to validate the VMM implementation, code and to ensure that it yields the desired results for known problems.

Mesh density effects and structured/unstructured mesh performance studies were also conducted. Figures $9,10,11,12$ and 13 demonstrate these studies.

\section{B. UEL validation - CTS, DNT and SEN3B tests}

Using the the VMM methodology described earlier crack propagation in laminated fiber reinforced composites was next undertaken. When through-the-thickness crack propagates in a laminated composite panel, a variety of failure mechanisms are initiated at the advancing primary crack which also shows a complex interaction between different mechanisms of failure. ${ }^{10}$ However, at some length scale, if we replace this advancing "crack like" feature by an advancing crack and an associated bridging zone, then it is of interest to examine whether the procedure that has been proposed here is able to reproduce the macroscopic details (the word macroscopic is intended to convey to the reader the scale at which the laminated panel is viewed 
as an appropriately homogenized monolithic structural panel) of the structural response. To this end, the proposal then is to examine whether the data obtained from the CTS and DNT tests (and in association with the VMM) is able to reproduce the mechanics (at the macroscopic scale) of an independent structural test (here this is the SEN3B test). The development of the crack is due to the softening of the highly stressed elements (Figure 14). These softened elements can be clearly seen in the magnified tension specimen deformed mesh (Figure 14(c)). Next, a similar study of the CTS tests were performed to extract the fracture energy input required for the VMM simulations. Figure 6 shows the specimen geometry and test setup for the CTS fracture tests. The load and load point displacement (between the loading rollers) was measured during the test. The fracture energy was calculated by dividing the total area under the load-displacement curve by the total crack length and thickness of the specimen. Figure 14(b) shows the deformed meshes of the SEN3B specimens. The observed crack progress was inline with the experimental observations. Figure 15 offers validation of the VMM implementation, as there is good agreement between the simulation results and experiments.

Our present implementation is able to predict the macroscopic response (load - displacement) with substantial accuracy. The crack path evolution is in agreement with the experimental observations for the problems that have been discussed here. However, for specimens which show crack propagation along arbitrary paths (as in, for example, eccentric loading of SEN3B specimens), the crack initiation and propagation conditions are currently being studied. Initial results indicate that a simple stress like measure for initiation yields results that are not in agreement with experimental observations.

The VMM results were also compared with the results from the traditional discrete cohesive zone model (DCZM) simulations which are widely applied to study crack advancement problems with crack bridging (Figure 16). Since, the DCZM method and other similar methods ${ }^{9,11-14}$ require a priori knowledge of crack path, these methods can only be used, at best, in situations where a good understanding of the failure process is a-priori known (such as in a stiffener separation from a panel, problem) but this is not necessary in the VMM implementation. This permits the application of the VMM method to a wide range of problems involving complex crack paths. However all the problems considered here involve straight crack propagation (mostly involving Mode I fracture), but a general problem will involve mode mixity with both Mode I \& Mode II fracture. We are currently extending the VMM implementation to treat mixed mode fracture of laminated composite panels with associated experimental verification and validation.

\section{Conclusion}

By treating a laminated fiber reinforced composite panel as an equivalent, orthotropic panel, we have demonstrated that the VMM based finite element implementation is capable of predicting the macroscopic mechanics of a fracturing laminated panel. The inputs to the VMM are similar to that which is needed for traditional cohesive zone model implementations of crack propagation predictions; a cohesive strength and an associated fracture energy. Using these two quantities as input, the mode I fracture of a laminated panel has been studied and its macroscopic response has been accurately captured. Extensions of the VMM to understand crack advancement under mixed mode conditions is currently being attempted and will be reported at future AIAA SDM conferences.

\section{References}

\footnotetext{
${ }^{1}$ Garikipati,K., "On Strong Discontinuities in Inelastic Solids and their Numerical Simulation", Ph.D. dissertation, Stanford University, California, 1996.

${ }^{2}$ Armero,F. \& Garikipati,K., "An Analysis of Strong Discontinuities in Multiplicative Finite Strain Plasticity and their Relation with the Numerical Simulation of Strain Localization in Solids", International Journal of Solids and Structures, 33, 2863-2885, 1996.

${ }^{3}$ Armero,F. "Large-scale modeling of localized dissipative mechanisms in a local continuum: application to the numerical simulation of strain localization in rate-dependent inelastic solids", Mechanics of Cohesive-Frictional Materials, 4, 101-131, 1999.

${ }^{4}$ Regueiro,R.A.\& Borja, R.I., "A finite element model of localized deformation in frictional materials taking a strong discontinuity approach", Finite Elements in Analysis and Design, 33, 283-315, 1999.

${ }^{5}$ Borja,R.I \& Regueiro,R.A., "Strain localization in frictional materials exhibiting displacement jumps", Computer methods in applied mechanics and engineering, 190, 2555-2580, 2001.
} 
${ }^{6}$ Garikipati,K., "A Variational Multiscale Method to Embed Micromechanical Surface Laws in the Macromechanical Continuum Formulation", Computer Methods in Engineering and Science, 3(2), 175-184, 2002.

${ }^{7}$ Simo,J.C., \& Rifai,M.S., "A class of mixed assume strain methods and the method of incompatible modes", International Journal of Numerical Methods in Engineering, 29, 1595-1638, 1990.

${ }^{8}$ Sun, C.T. \& Chen, J.L., "A Simple Flow Rule for Characterizing Nonlinear Behavior of Fiber Composites", Journal of Composite Materials, 23, 1009-1020, 1989.

${ }^{9}$ Gonzales Ruiz, Anna Pandolfi \& Ortiz, M., "Three-dimensional cohesive modelling of dynamic mixed-mode fracture", International Journal for Numerical Methods in Engineering, 52, 97-120, 2001.

${ }^{10}$ Luis Gonzales \& Knauss, W.G., "Scaling global fracture behavior of structures-sized laminated composites", International Journal of Fracture, 118, 363-394, 2002.

${ }^{11}$ Zhou, F. \& Molinari, J.F., "Dynamic crack propagation with cohesive elements: a methodology to address mesh dependency", International Journal for Numerical Methods in Engineering, 59, 1-24, 2003.

${ }^{12}$ Jin, Z.H. \& Sun, C.T., "Cohesive zone modeling of interface fracture in elastic bi-materials", Engineering Fracture Mechanics, 72, 1805-1817, 2005.

${ }^{13} \mathrm{Li}, \mathrm{S} .$, Thouless, M.D., Waas, A.M., Schroederd, J.A. \& Zavattierid, P.D., "Use of a cohesive-zone model to analyze the fracture of a fiber-reinforced polymermatrix composite”, Composites Science and Technology, 65, 537-549, 2005.

${ }^{14}$ Xie, D. \& Waas, A.M., "Discrete cohesive zone model for mixed-mode fracture using finite element analysis", Engineering Fracture Mechanics, 73 (13), 1783-1796, 2006.

${ }^{15}$ Stakgold,I. Boundary Value Problems in Mathematical Physics, Macmillian, New York, 1968. 


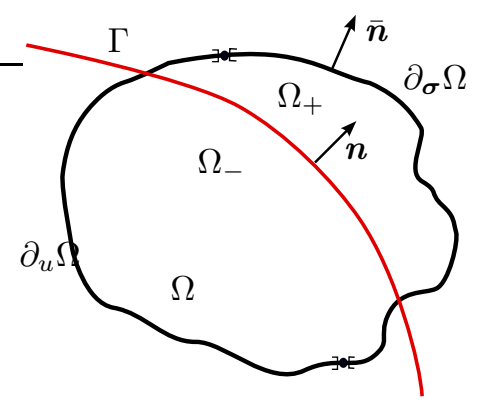

Figure 1. Continuum body in the presence of a displacement discontinuity

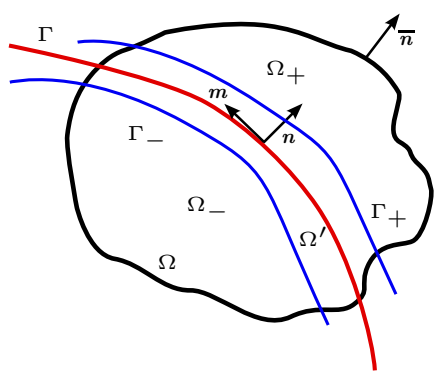

Figure 2. Decomposition of continuum body into region where coarse scale and fine scale displacements are defined
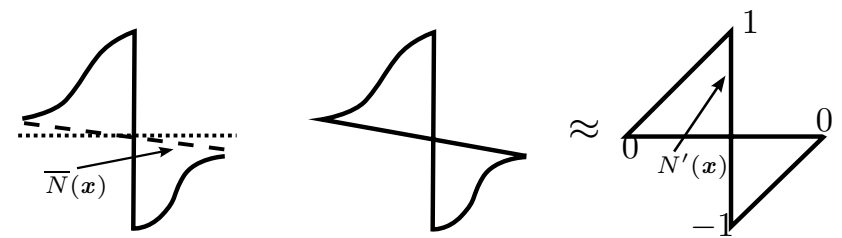

Figure 3. Coarse and fine scale interpolation functions in one dimensional setting.

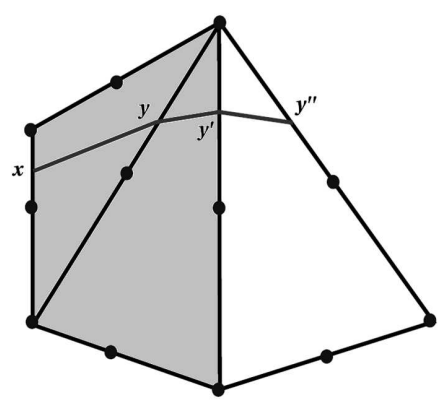

Figure 4. Band tracking algorithm 


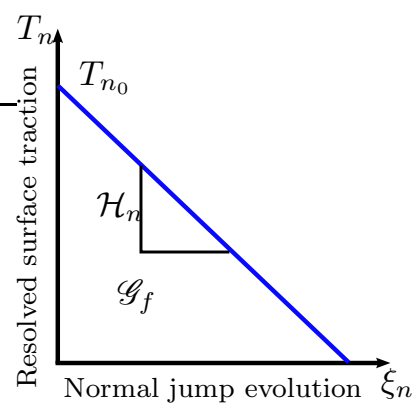

Figure 5. Linear softening response for micro-mechanical surface

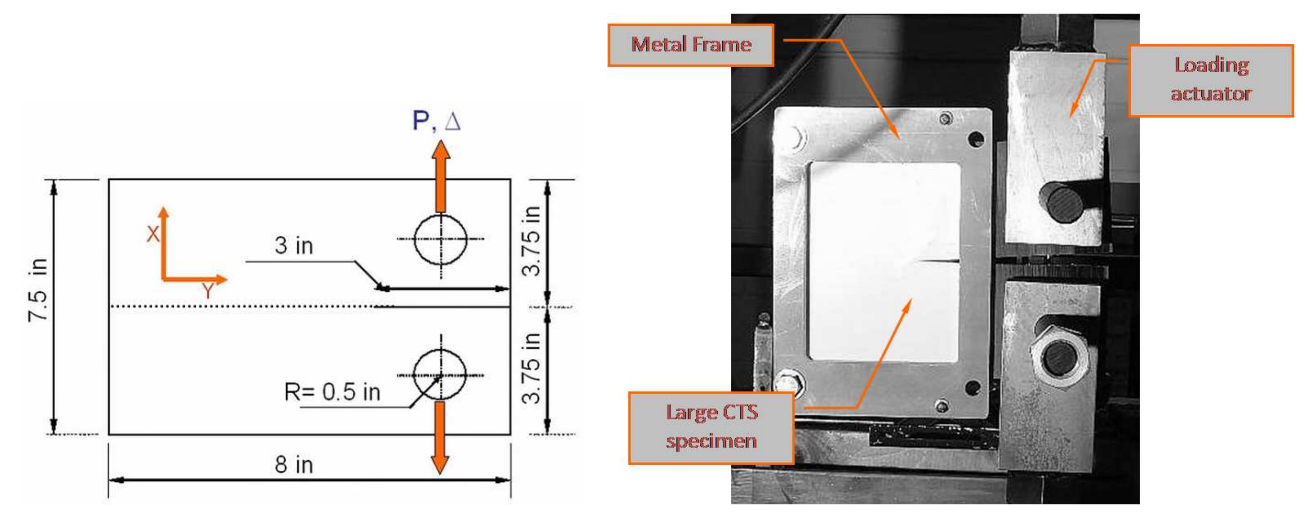

(a)

(b)

Figure 6. Fracture toughness values are obtained by Compact Tension(CT) specimen experiments. a)CT specimen geometry b)Experimental setup.

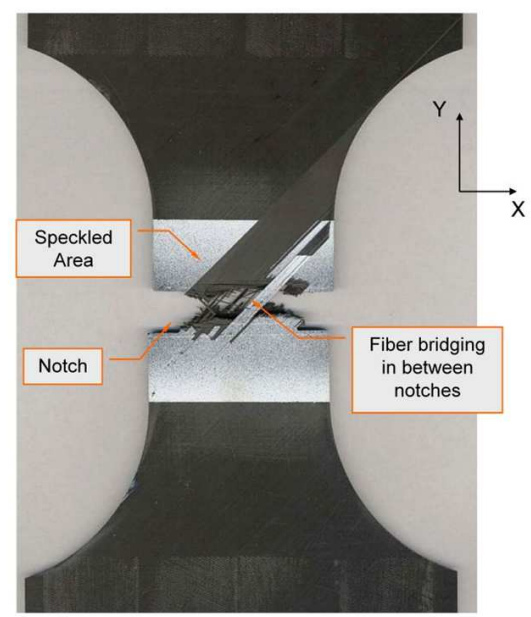

Figure 7. Critical localization stress value obtained by double notch tension specimen experiments. Shown here is a post failure specimen 


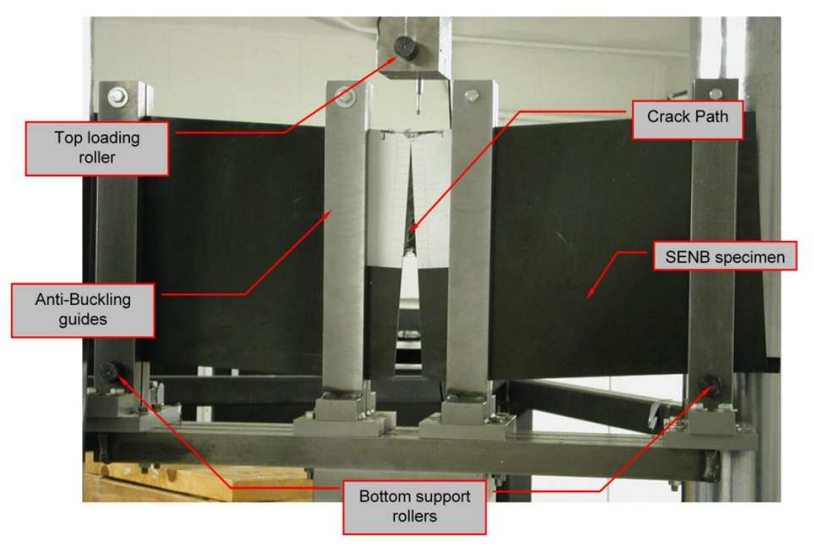

Figure 8. Single Edge Notch Bending(SENB) specimen experimental setup. The SENB results are used for validating the VMM simulation results.

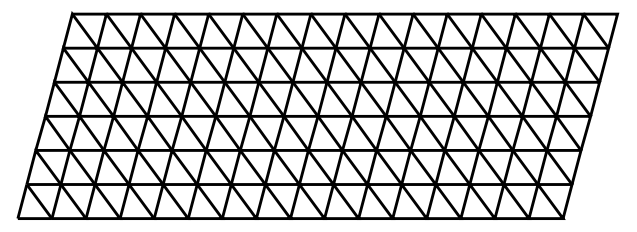

(a)

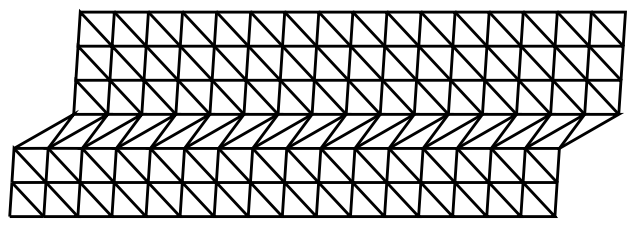

(b)

Figure 9. Deformed shape of block undergoing uniform shear load, (a) uniform deformation prior to localization, (b) highly localized deformation following the development of the displacement discontinuity.

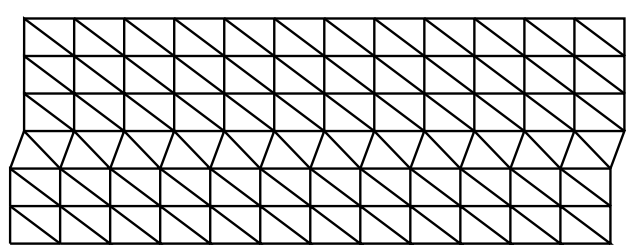

(a)

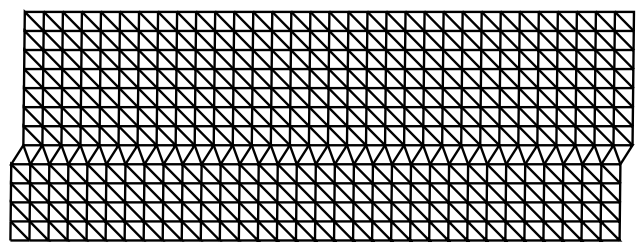

(b)

Figure 10. Structured mesh at different levels of refinement, (a) 144 elements, (b) 768 elements. 


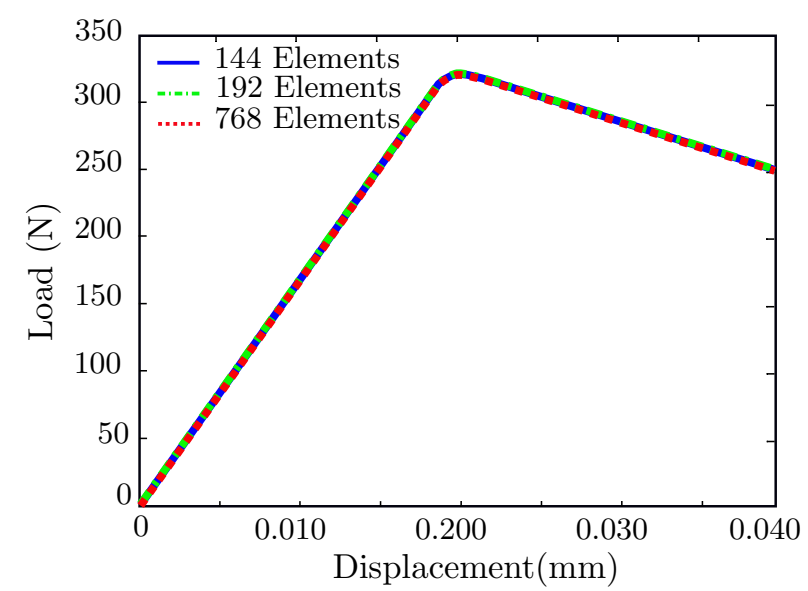

Figure 11. Load-displacement curves for various levels of mesh refinement (structured meshes).

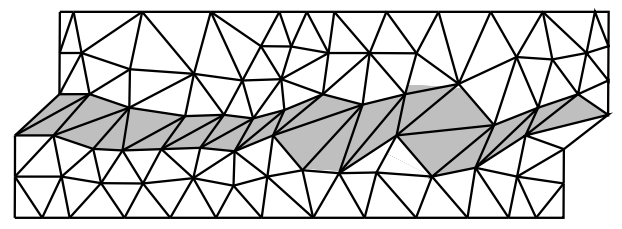

(a)

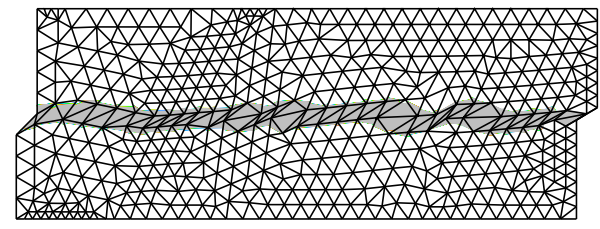

(b)

Figure 12. Deformed configuration of unstructured meshes due to shear loading, (a) 128 elements, (b) 931 elements.
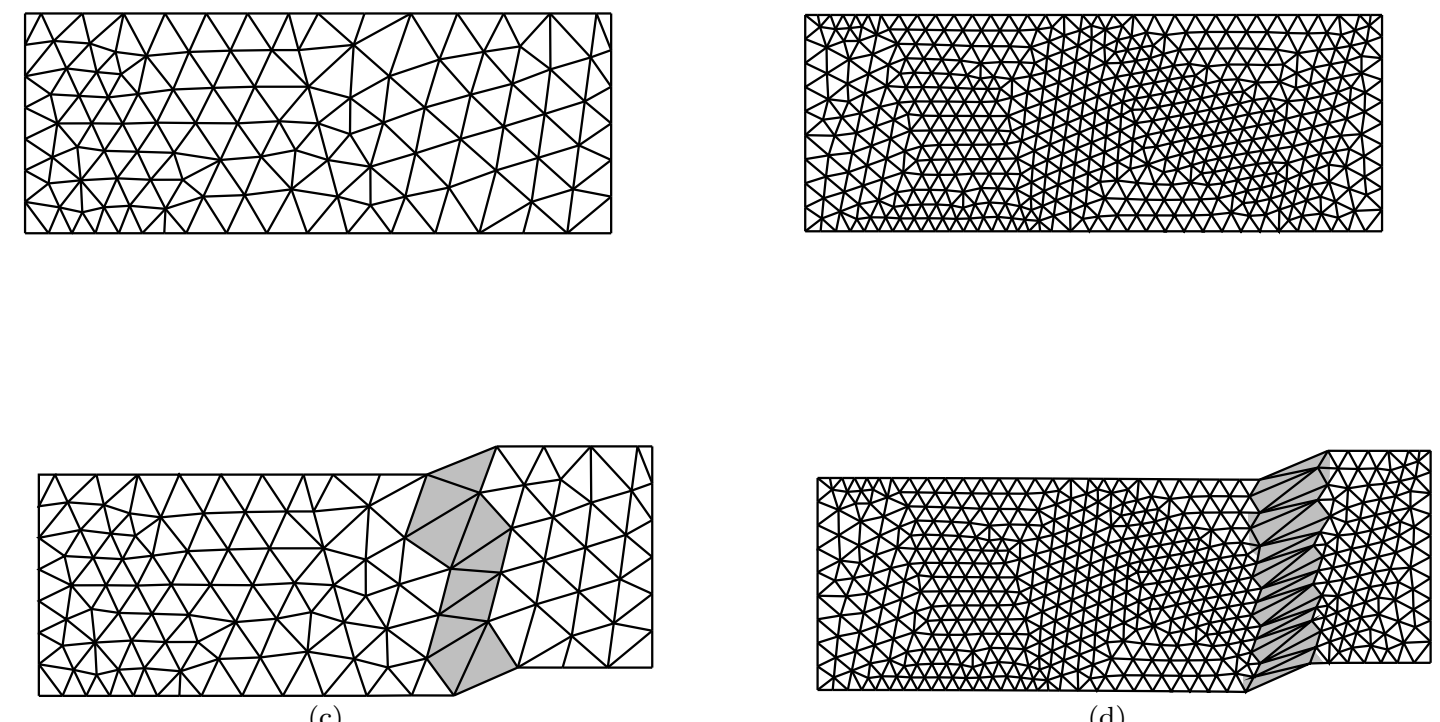

(d)

Figure 13. Undeformed and deformed configuration of unstructured meshes due to tensile loading. (a) undeformed 197 elements, (b) undeformed 1010 elements, (c) deformed 197 elements, (d) deformed 1010 elements. 


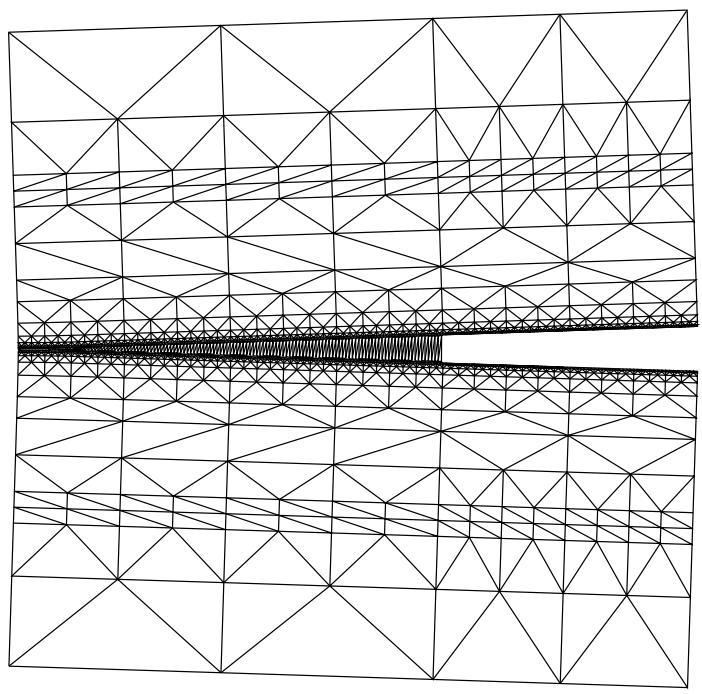

(a)

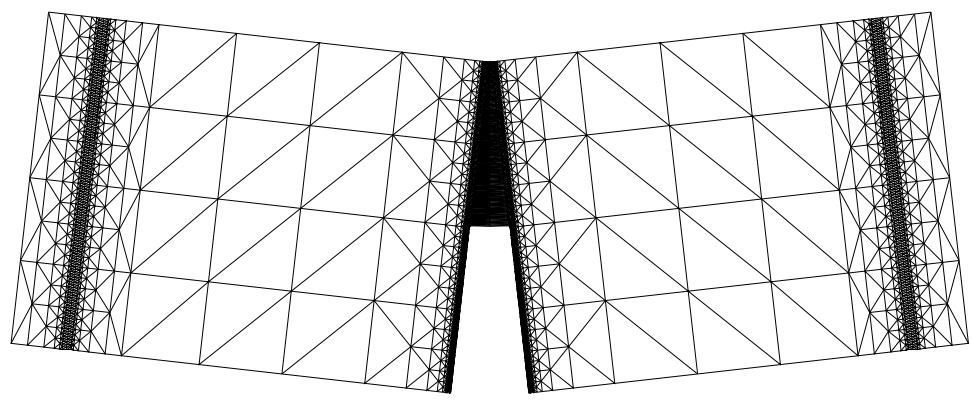

(b)

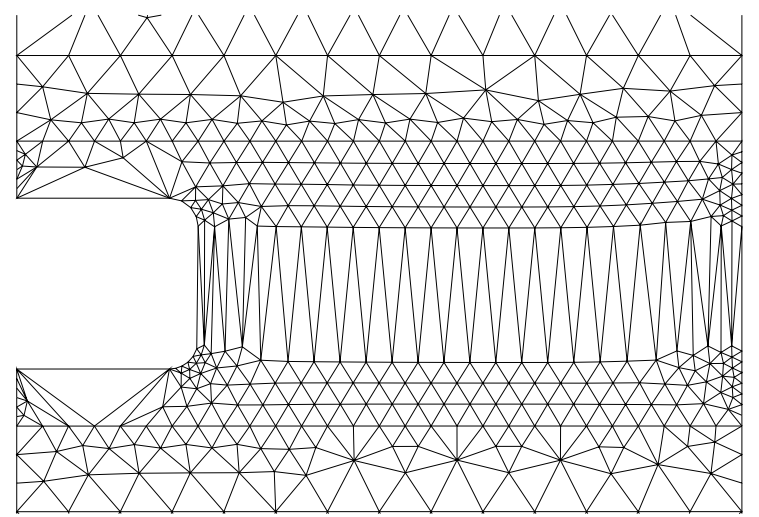

(c)

Figure 14. Deformed configuration of standard fracture specimen meshes: (a) CT specimen, (b) SENB specimen, (c) Tension specimen (magnified to show softened elements). 


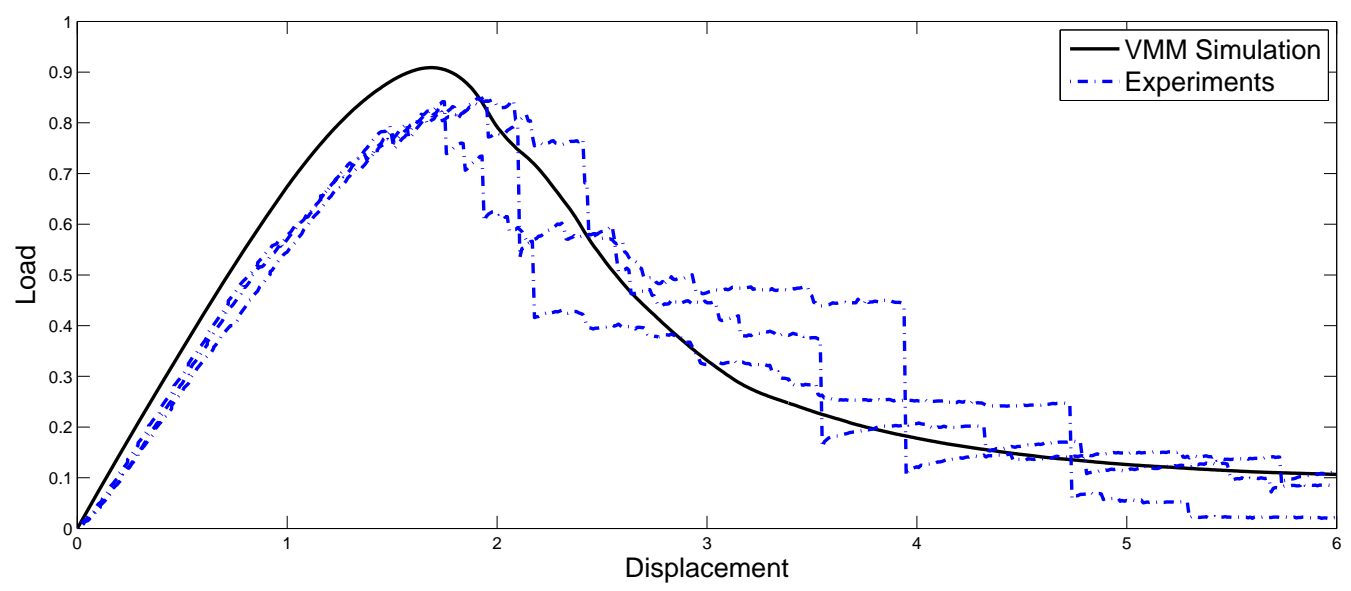

Figure 15. Comparison of VMM simulation results with experimental results for SENB specimen. Experimental test data from three tests are shown.

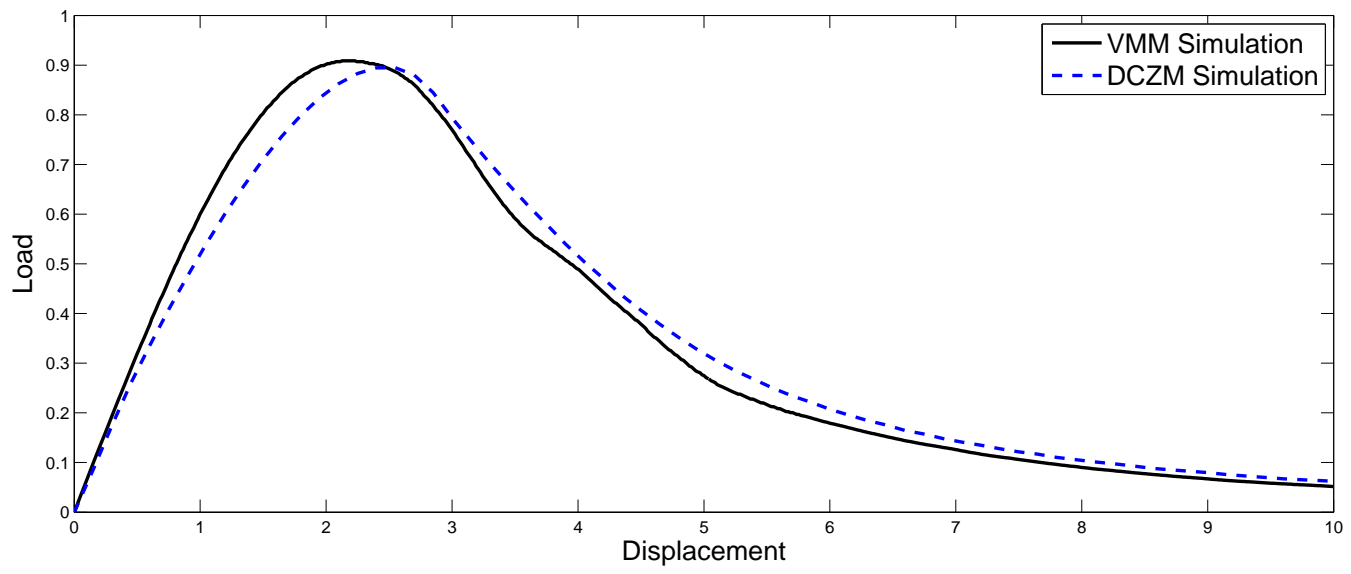

Figure 16. Comparison of VMM and Discrete Cohesive Zone Method(DCZM) simulation results for CT specimen. 